ESAIM: PROCEEDINGS, February 2007, Vol.16, 16-32

Eric Cancès \& Jean-Frédéric Gerbeau, Editors

DOI: $10.1051 /$ proc:2007007

\title{
DIRECT NUMERICAL AEROACOUSTICS FOR LOW MACH NUMBERS USING ONE UNSTRUCTURED GRID
}

\author{
A. Gordner ${ }^{1}$ And G. WitTum ${ }^{1}$
}

\begin{abstract}
Using an unstructured grid, for which the mesh size is adapted to the local flow and acoustic solution, one can reduce the number of unknowns needed for a direct aeroacoustic simulation. The compressible Navier-Stokes equations for Mach numbers $0<M<1$ are considered. However, the unstructured grid together with the increasing stiffness of the problem for $M \rightarrow 0$ reveals new numerical challenges. One is, to find an optimal time step size, that will reveal an equal numerical error distribution on the computational domain. Therefore, CFL-numbers greater than 1 are required, so that an implicit approach is chosen to overcome this complexity. The multigrid method used to solve the resulting algebraic system of equations is robust against the Mach number. Hence, the convergence rate does not deteriorate for $M \rightarrow 0$. The accuracy of the time and space discretisation is investigated with respect to their numerical dispersion and diffusion properties. Furthermore, the order of the space discretisation is increased with a $\tau$-extrapolation method, which is embedded into the multigrid procedure. The accuracy of the method and the reduction of unknowns is demonstrated by numerical simulations.
\end{abstract}

\section{INTRODUCTION}

Aeroacoustic issues become more and more important in technical applications especially in situations, where acoustic has also a considerable impact on the overall flow solution. However, from the scientific point of view, fully coupled, direct aeroacoustic numerical simulations with the compressible Navier-Stokes equations can model the physical properties quite well, but suffer under numerical complexities for Mach numbers $0<M<1$. In space, a multiscale solution with different acoustic and flow wave lengths is expected in this Mach number regime. In addition the underlying Navier-Stokes equations become stiff, which affects the required time step size.

To overcome the numerical difficulties, most often numerical procedures decouple the acoustic from the fluid flow scales, neglecting the acoustic impedance. In these hybrid approaches the flow is simulated independently from the acoustic pressure generation and distribution. Afterwards, acoustic source terms and the distribution of the acoustic waves in and into the far-field are calculated on the basis of the flow solution. A wide variety of different methods can be found in literature, see Lele [14] and Költsch [13].

Another way to overcome the stiffness of the problem is preconditioning, i.e. Lynn [15], Turkel [27]. By preconditioning, the acoustic eigenvalues, responsible for the stiffness, will be down scaled. Hereby, the natural impedance condition of acoustic waves and their velocity of propagation is artifically changed, so that time accuracy is lost. For stationary problems and for problems in which the acoustic is neglected i.e. Guillard et al. [11], Bijl et al. [4], Turkel [27], this is a well known method. One can preserve time accurateness using dual time stepping schemes, see i.e. Turkel et al. [28].

${ }^{1}$ Institut für Informatik, Technische Simulation, Universität Heidelberg, Im Neuenheimer Feld 368, Germany

(c) EDP Sciences, SMAI 2007 
Using one-grid and the full compressible Navier-Stokes equations, the acoustic impedance condition and the full coupling between flow and acoustic can be preserved, but one has to deal with additional numerical challenges. A possible solution method is presented here.

Let the compressible Navier-Stokes equations be given in primitive variables on the domain $\Omega$ with appropriate boundary conditions for pressure, density and velocity $\left.\mathbf{q}\right|_{\delta \Omega}=\left.\{\rho, \mathbf{v}, p\}\right|_{\delta \Omega}$. The equations are normalised by given reference values $\mathbf{q}_{r e f}=\left\{\rho_{\text {ref }}, \mathbf{v}_{\text {ref }}, p_{\text {ref }}\right\}$ and length $L$. The reference Mach number $M=\frac{\left|\mathbf{v}_{r e f}\right|}{a_{r e f}}$ appears in convection terms, due to the non-dimensionalisation by a reference time $t_{r e f}=\frac{L}{a_{r e f}}$, which is based on the reference speed of sound $a_{r e f}=\sqrt{\frac{p_{r e f}}{\rho_{r e f}}}$. The conservation equations for mass, momentum and energy under the assumption of a caloric ideal gas with the equation of state $p=(\gamma-1) \rho e$, can be written in the following form:

$$
\begin{gathered}
\frac{\partial \rho}{\partial t}+M \nabla \cdot(\rho \mathbf{v})=0 \\
\frac{\partial \rho \mathbf{v}}{\partial t}+M \nabla \cdot\left(\rho \mathbf{v} \otimes \mathbf{v}^{T}\right)=-\frac{1}{\gamma M} \nabla p+\frac{M}{R e}(\nabla \cdot \tau) \\
\frac{1}{\gamma-1} \frac{\partial p}{\partial t}+\frac{\gamma M}{\gamma-1} \nabla \cdot(p \mathbf{v})=-\frac{\gamma M^{2}}{2}\left(\frac{\partial \rho \mathbf{v}^{2}}{\partial t}-M \nabla \cdot\left(\rho \mathbf{v}^{2} \mathbf{v}\right)\right)+\frac{\gamma M^{3}}{R e} \nabla \cdot\left(\tau^{T} \mathbf{v}\right),
\end{gathered}
$$

with $M$ the global reference Mach number, Re the Reynolds number, $\tau$ the stress tensor and $\gamma=1.4$ the adiabatic coefficient for a caloric ideal gas.

We restrict ourselves to smooth solution with Mach numbers

$$
0<\epsilon<M=\frac{H e}{S t}<\delta
$$

$H e=\frac{L f}{a_{r e f}}$ denotes the global Helmholtz number, $S t=\frac{L f}{\mid \mathbf{v}_{\mathbf{r e f}}}$ is the global Strouhal number for given frequency $f$.. Since we are interested in acoustic compact domains, the value $\epsilon$ is greater than zero and the Helmholtz number and Strouhal number fulfill the condition $S t>H e \simeq 1 . \delta$ is chosen small enough, to prevent acoustic driven or flow driven shocks and discontinuous solutions.

For decreasing Mach number $M$, the speed of sound becomes larger in comparison to the convection speed. Since the frequency of the far-field acoustic pressure $f_{a}$ and the near-field dynamic pressure $f_{v}$ are identical, the wave lengths vary corresponding to the different transport velocities of the waves and one ends up in a multiscale problem. Hence, the local ratio between the acoustic wave length $\lambda_{a}$ and the dynamic pressure wave length $\lambda_{v}$, that causes the acoustics, is proportional to the local ratio between the convection speed $v$ and the speed of sound $a$, and therefore proportional to the local Mach number $M_{l}$

$$
f_{a}=f_{v}, \quad \Longrightarrow \quad \frac{\lambda_{v}}{\lambda_{a}}=\frac{v}{a}=M_{l}
$$

Thus, if one needs \#p points per wave length to resolve an acoustic wave or a fluid flow wave with numerical errors less than $\sigma$, one would need a coarser grid in the acoustic far-field, than in the near fluid flow field.

Under the assumption, that the reference values for velocity and speed of sound are chosen in such a way that

$$
\begin{aligned}
& v_{\text {ref }}=\max _{x \in \Omega, t \in \mathbb{R}^{+}}|\mathbf{v}(x, t)|, \\
& a_{\text {ref }}=\min _{x \in \Omega, t \in \mathbb{R}^{+}}|a(x, t)|,
\end{aligned}
$$

the relation for the local Mach number $M_{l}(x, t)=\frac{\lambda_{v}}{\lambda_{a}} \leq M$ holds for all $x \in \Omega$ and $t \in \mathbb{R}^{+}$. Thus, the local wave length of an acoustic far-field wave would be always greater than 


$$
\lambda_{a} \geq \frac{\lambda_{v}}{M} .
$$

Therefore, the ratio between the acoustic far-field mesh size $h_{a}$ and the near flow field mesh size $h_{v}$ of the computational grid can be defined as

$$
h_{a}=\frac{h_{v}}{M} .
$$

This is valid under the assumption, that the discretisation error in the acoustic far-field in comparison to the near flow field is of the same size or better.

Remark 1.1. We are just considering h-adaptivity. For hp-adaptivity, things become more complicated since then the number of points needed per wave length can differ as well.

This is the basic idea of one unstructured grid, for which the local mesh size is adjusted according to the expected wave length solution in order to guarantee an equally distributed numerical error in space. In the next section an estimate is given how much grid points can be saved in comparison to an equally spaced grid.

For small Mach numbers, the highly unstructured grid and the increasing stiffness of the problem, give rise to a new question. The choice of an optimal time step size and time discretisation. Explicit time discretisation methods would require an extreme small time step to fulfill the CFL-condition on the unstructured grid. It will be shown in section 3.1, that for an equally distributed numerical error $C F L$-numbers proportional to $C \cdot \frac{1}{M}$ are required. Hence, an implicit time discretisation method is used. The constant $C$ depends on the accuracy of both, the time and space discretisation.

Another aspect important for time-accurate aeroacoustic simulations, is the accuracy of the time and space discretisation in use with respect to numerical damping and dispersion errors. An implicit Runge-Kutta method, the three stage Fractional Step method, is applied and investigated with respect to its numerical diffusion and dispersion errors. For space discretisation a Finite Volume approach on collocated grids is used, which is of 2nd order accuracy for acoustics waves. Higher order Finite Volume approaches for collocated grids are very costly. Hence, an alternative approach is shown, where higher order solutions in space can be achieved by an extrapolation method, as long as the solutions are smooth. The extrapolation can be included into the multigrid procedure, which is known as $\tau$-extrapolation. In addition, the multigrid solver is applied to solve iteratively the resulting algebraic system of equations. The basic principles of a $\tau$-extrapolation are presented in section 3.2.1.

In section 4 the one-grid approach and the presented numerical procedure is applied to a travelling wave configuration at Mach number $M=10^{-3}$, for which the numerical properties of the proposed methods are tested. In case of a trailing edge thickness noise configuration of a flat plate the benefit of an unstructured grid is illustrated for $M=0.2$.

\section{THE ONE-GRID APPROACH}

In order to break the numerical complexity, the mesh size on an unstructured grid can be optimized to end up with an equally accurate physical behaviour of the solution on the whole domain with respect to numerical diffusion and dispersion errors. The grid in the acoustic far-field can be made coarse, whereas other parts of the domain $\Omega$ have to be fine enough to resolve the small scale velocity perturbations in the near flow field, which generate the acoustic modes, see Figure 1.

The numerical diffusion and dispersion errors of the space discretisation are assumed to be proportional to the wavenumber of the solution and depending on the number of grid points $\# p$ used per wave length respectively.

An optimal ratio between the far-field grid size $h_{a}$ and the near flow-field grid size $h_{v}$ on an ideally unstructured grid would lead to an almost constant discretisation error in space on the domain $\Omega$. The grid size ratio is given by equation (8) and depends on the global Mach number $M$ only. For $h$-adaptivity, the ratio between the smallest grid size $h_{\min }$ and the largest grid size in the acoustic far-field $h_{\max }$ would be 


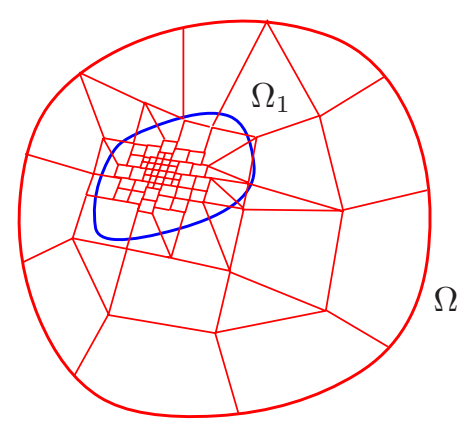

FiguRE 1. One-grid direct approach with locally unstructured grid. To identify the near flow field $\Omega_{1}$, the border of $\Omega_{1}$ is plotted.

$$
M=\frac{h_{\min }}{h_{\max }} .
$$

Now the amount of unknowns that can be saved with an unstructured grid in comparison to an equally spaced grid with mesh size $h_{\min }$ are estimated. If the dimension of the problem is $d$, then the number $\# n$ of grid nodes can be estimated with

$$
\# n(M)=\frac{\left|\Omega_{1}\right|}{h_{\min }^{d}}+\frac{|\Omega|-\left|\Omega_{1}\right|}{h_{\max }^{d}}
$$

Using equation (9), one can derive the ratio between the number of nodes needed on an equally spaced grid $\left(\Omega_{1}=\Omega\right.$ and $\left.M=1\right)$ and the number of nodes needed on an unstructured grid as

$$
\frac{\# n(M)}{\# n(1)}=\left(1-M^{d}\right) \frac{\left|\Omega_{1}\right|}{|\Omega|}+M^{d}
$$

Now, let the fluid flow structures be given on $\Omega_{1}$ and remaining constant with varying Mach number. Since the domain $\Omega$ should be acoustical compact, $\Omega$ has to be increased with respect to $\Omega_{1}$ for $M \rightarrow 0$. Hence $\frac{\left|\Omega_{1}\right|}{|\Omega|} \sim C M^{d}$ can be expected, so that equation (11) becomes

$$
\frac{\# n(M)}{\# n(1)}=\left(1-M^{d}\right) C M^{d}+M^{d} \approx C M^{d} .
$$

Thus, the benefit is Mach number-dependent.

\section{Discretisation}

An optimal discretisation would lead to an constant numerical error distribution on the computational domain. I.e. in the case of a multiscale coupled problem which is the case for direct numerical aeroacoustics for $M<1$, it does not pay-off to increase the accuracy in one part of the domain. In the first section the optimal time step size is determined to make a decision between explicit or implicit time discretisation. For time accurate aeroacoustic simulations, numerical errors have to be identified and controlled, so that they are smaller than a desired error bound $\sigma$. In section 3.2 the discretisation in time and space will be illustrated and analysed with respect to their numerical dispersion and diffusion errors. First, the question how the optimal time step size looks like will be investigated. 


\subsection{Time discretisation}

An optimal time step size used on the whole domain $\Omega$ is sought. Methods that are using local time stepping on different parts of the domain $\Omega$, i.e. Roller et al. [18], Munz et al. [17], are not considered here.

An optimal choice for the time step size in correlation with the unstructured mesh size in the acoustic far-field and the near flow field would lead to a constant cut-off frequency $f_{\text {max }}$ on the whole domain $\Omega$. Hence, any frequency in the velocity field $f_{V}<f_{\max }$ and in the acoustic field $f_{A}<f_{\max }$ can be resolved with dispersion and diffusion errors in time and space lower than $\sigma$.

With the local CFL-number

$$
\operatorname{CFL}(x, \Delta t)=\frac{a \Delta t}{h(x)}, \quad x \in \Omega,
$$

their minimum and maximum value on the domain $\Omega$ for a given $\Delta t$ are given as

$$
C F L_{\max }(\Delta t)=\frac{a \Delta t}{h_{\min }}, \quad C F L_{\min }(\Delta t)=\frac{a \Delta t}{h_{\max }} .
$$

If one assumes the cut-off frequency $f_{\text {tmax }} \sim \frac{1}{C_{t} \Delta t}$ and the maximum cut-off wave length to be $\lambda_{h \max } \sim$ $C_{h} h_{\max }$, then one gets a different cut-off frequency $f_{\max }$ for the acoustic far-field in $\Omega$ and the near flow field in $\Omega_{1}$

$$
f_{\max _{V}}=\min \left(\frac{a}{C_{t} h_{\max } C F L_{\min }}, \frac{a}{C_{h} h_{\max }}\right)=\min \left(\frac{|\mathbf{v}|}{C_{t} h_{\min } C F L_{\min }}, \frac{|\mathbf{v}|}{C_{h} h_{\min }}\right)
$$

while for the acoustics in the near flow field domain $\Omega_{1}$

$$
f_{\max _{A}}=\min \left(\frac{a}{C_{t} h_{\min } C F L_{\max }}, \frac{a}{C_{h} h_{\min }}\right)
$$

holds. The constants $C_{t}$ and $C_{h}$ are depending on the order of the time and space discretisation and will not be further specified. The better the accuracies of the discretisations are, the smaller these constants will become. If the order of both methods are the same, both constants do not differ too much, such that $C_{G}=\frac{C_{h}}{C_{t}} \approx 1$. With equation (9), (14) and for a given time-step $\Delta t$, the local CFL-number varies like

$$
C F L(x, \Delta t) \in\left[C, \frac{C}{M}\left[\quad \forall x \in \Omega, \quad C \in \mathbb{R}^{+} .\right.\right.
$$

We put equation (17) into equations (15), (16) and replace $C_{h}$ with $C_{G} \cdot C_{t}$ and get

and

$$
f_{\max _{V}}=\min \left(\frac{a}{C_{t} h_{\max } C}, \frac{a}{C_{h} h_{\max } C_{G}}\right)
$$

Now, it can be derived

$$
f_{\max _{A}}=\min \left(\frac{a}{C_{t} h_{\max } C}, \frac{a}{C_{h} h_{\max } M C_{G}}\right)
$$

$$
\begin{array}{cl}
f_{\max _{V}}=f_{\text {max }_{A}}: & C>C_{G} \\
f_{\max _{V}}=\frac{C}{C_{G}} f_{\text {max }_{A}}: & M C_{G}<C \leq C_{G} \\
f_{\max _{V}}=M f_{\max _{A}}: & C \leq M C_{G} .
\end{array}
$$

With this result, the advantage of explicit or implicit time discretisation methods can be further examined. According to equation (17), for explicit methods $C$ has to be chosen such that $\frac{C}{M}<1$. Then, the optimal 
condition, where the cut-off frequency $f_{\max }=f_{\max _{V}}=f_{\max _{A}}$ is almost constant on the domain $\Omega$, is only fulfilled for $C_{G}<M$. This means that the order/accuracy of the space discretisation should be at least better by the factor $M$ than those of the explicit time discretisation. In all other cases, i.e. when time and space discretisations are of the same order of accuracy $C_{G} \approx 1$, or the order/accuracy of the time discretisation is better than that of the space discretisation $C_{G}>1$, for explicit methods the relation $f_{\max _{V}} \leq M f_{\max _{A}}$ holds. This means, that for $M<1$ a smaller time step size required for an explicit method does not cause a higher accuracy on the whole domain $\Omega$. I.e. if $C_{G} \approx 1$, one would need a $M$-times lower time step size for the explicit method than for the implicit method, but would gain the same cut-off frequency and the same quality of the solution.

Considering equation (20), $C$ has to be chosen $C \approx C_{G}$ to get the same cut-off frequency on the whole domain. This results into local CFL-numbers greater than 1, which advantage implicit methods for very small Mach numbers $M<<1$.

Remark 3.1. For moderate low Mach numbers $0.1<M<1$ the use of explicit methods would lead only to a small unbalanced cut-off frequency distribution. It will become severe for very small Mach numbers, $0<M<<1$.

\subsection{Accuracy of time and space discretisation}

A fully implicit approach is considered here, where also the convection terms of the compressible Navier-Stokes equations are treated implicitly.

The Navier-Stokes equations given in (1), (2) and (3) can be written in the form

$$
\frac{\partial M\left(\mathbf{q}_{\mathbf{c}}, x, t\right)}{\partial t}+A\left(\mathbf{q}_{\mathbf{c}}, x, t\right)=F\left(\mathbf{q}_{\mathbf{c}}, x, t\right)=0
$$

with the mass operator $M$ and the stiffness operator $A$ of the partial differential equations and $\mathbf{q}_{\mathbf{c}}=f(\mathbf{q})$ is given as $\mathbf{q}_{\mathbf{c}}=\{\rho, \rho \mathbf{v}, p\}$.

The nonlinear iteration with counter $i$ can be written in the following form

$$
\begin{aligned}
\frac{\partial F\left(\mathbf{q}_{\mathbf{c}}^{\mathbf{i}}, x, t\right)}{\partial \mathbf{q}} \quad \mathbf{c} & =-F\left(\mathbf{q}_{\mathbf{c}}^{\mathbf{i}}, x, t\right) \\
\mathbf{q}_{\mathbf{c}}^{\mathbf{i}+\mathbf{1}} & =\mathbf{q}_{\mathbf{c}}^{\mathbf{i}}+\mathbf{c}
\end{aligned}
$$

or given in a more abstract form

$$
\begin{aligned}
& J\left(\mathbf{q}^{\mathbf{i}}\right) \mathbf{c}=b\left(\mathbf{q}^{\mathbf{i}}\right) \\
& \mathbf{q}^{\mathbf{i}+\mathbf{1}}=\mathbf{q}^{\mathbf{i}}+\mathbf{c} .
\end{aligned}
$$

The Jacobian $\frac{\partial F\left(q_{c}, x, t\right)}{\partial q}$ is derived with respect to the primitive variables $\mathbf{q}=\{\rho, \mathbf{v}, p\}$. If the Jacobian exists and the iteration converges, there is no difference in the result obtained with the Jacobian built with respect to conservation variables $\mathbf{q}_{\mathbf{c}}$. However, a detailed analysis is beyond the scope of the paper.

Time discretisation is done with a 3 stage stiffly accurate diagonal implicit Runge-Kutta scheme, the Fractional Step method, introduced by Glowinski [9] and described in Turek [26]. The Butcher type matrix scheme is given in equation (24). 


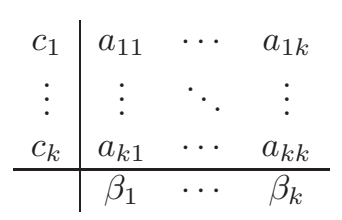

\begin{tabular}{c|cccc}
0 & 0 & 0 & 0 & 0 \\
$\alpha$ & $1-3 \alpha$ & $4 \alpha-1$ & 0 & 0 \\
$1-\alpha$ & $1-3 \alpha$ & $1-2 \alpha$ & $4 \alpha-1$ & 0 \\
1 & $1-3 \alpha$ & $1-2 \alpha$ & $\alpha$ & $4 \alpha-1$ \\
\hline & $1-3 \alpha$ & $1-2 \alpha$ & $\alpha$ & $4 \alpha-1$
\end{tabular}

The method is of second order for $\alpha=1-\frac{1}{2} \sqrt{2}$, see Müller-Urbanik [16]. To analyse the method with respect to its dispersion and diffusion accuracy, the Laplace-transformation method introduced by Tam \& Webb [29] is adjusted to the non-equal time step sizes of the implicit Runge-Kutta method. More details can be found in Gordner [10].

A linear model equation of the form

$$
\frac{\partial f(t)}{\partial t}+i \hat{\omega} f(t)=0 \text { with } f(0)=1, \quad \hat{\omega} \in \mathbb{C}, R e(\hat{\omega})>0
$$

with the solution $f(t)=\left\{\begin{array}{r}e^{-i \widehat{\omega} t}, t \geq 0 \\ 0, t<0\end{array}\right.$ is used.

The Laplace-transformation

$$
\tilde{f}(i \omega)=\mathcal{L}(f(t))=\int_{0}^{\infty} f(t) e^{-i \omega t} d t, \omega \in \mathbb{C}
$$

into the frequency space $i \omega$ reveals the accuracy of the approximated discrete eigenvalue $i \widehat{\omega}$.

According to the Butcher scheme (24), in each $k$ th $\Theta_{k}$-step of the Runge-Kutta method - ( $k$ th row of the Butcher scheme) -, following equation has to be solved

$$
f(t)-f\left(t-\left(c_{k}-c_{k-1}\right) \Delta t\right)=\Delta t \sum_{j=1}^{k}\left(a_{k j}-a_{(k-1) j}\right) \frac{\partial f\left(t-\left(c_{k}-c_{k-(j-1)}\right) \Delta t\right)}{\partial t}
$$

The time derivative is substituted by the model equation (25) and the Laplace-transformation of the given continuous solution of the model equation using the translation theorem reveals the characteristic equation of each $k$ th $\Theta$-step, which can be written in the form

$$
\widehat{\omega} \Delta t=\frac{i\left(1-e^{\left(c_{k}-c_{k-1}\right) i \omega \Delta t}\right)}{\sum_{j=1}^{k}\left(a_{k j}-a_{k-1 j}\right) e^{\left(c_{k}-c_{k-(j-1)}\right) i \omega \Delta t}} .
$$

Each numerical solution of the $k$ th sub-step of the Runge-Kutta method $e_{k}^{i \omega t}$ has to fulfill the characteristic equation (27), which gives a relation between the discrete $\omega \Delta t$ and the continuous $\widetilde{\omega} \Delta t$. The difference in the real part $R e(\omega \Delta t)-R e(\widehat{\omega} \Delta t)$ corresponds to a dispersion error, while the difference in the imaginary part $\operatorname{Im}(\omega \Delta t)-\operatorname{Im}(\widehat{\omega} \Delta t)$ indicates a damping error.

Each of the three $\Theta_{i}$-substeps of the Runge-Kutta scheme is analysed separately. The $\omega \Delta t$ of the entire scheme is the weighted sum of the three sub-steps

$$
\begin{gathered}
\operatorname{Re}(\omega \Delta t)_{\text {gesamt }}=\alpha \operatorname{Re}\left((\omega \Delta t)_{\Theta_{1}}+(1-2 \alpha) \operatorname{Re}\left((\omega \Delta t)_{\Theta_{2}}+\alpha \operatorname{Re}\left((\omega \Delta t)_{\Theta_{3}}\right.\right.\right. \\
\operatorname{Im}(\omega \Delta t)_{\text {gesamt }}=\alpha \operatorname{Im}\left((\omega \Delta t)_{\Theta_{1}}+(1-2 \alpha) \operatorname{Im}\left((\omega \Delta t)_{\Theta_{2}}+\alpha \operatorname{Im}\left((\omega \Delta t)_{\Theta_{3}}\right.\right.\right.
\end{gathered}
$$

respectively.

In Figure 2, the real and imaginary part of $\omega \Delta t$ are plotted against $\widetilde{\omega} \Delta t$ for $\widetilde{\omega} \in \mathbb{R}^{+}$. Herein, the Fractionalstep method is compared with the standard 4th order explicit Runge-Kutta method. The Butcher scheme for the explicit method can be found i.e. in Ferziger [7]. 


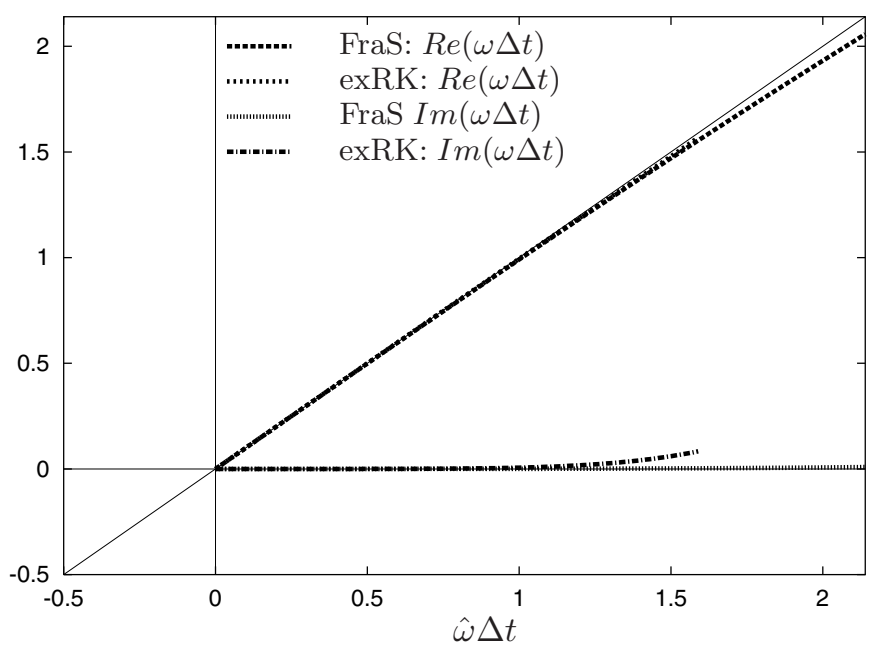

Figure 2. Numerical diffusion and dispersion error of the Fractional-Step method compared to the standard explicit 4th order Runge-Kutta scheme.

It is illustrated, that the 2nd order Fractional-Step method has almost the same properties with respect to dispersion and diffusion errors as a 4th order explicit Runge-Kutta method. For a dispersion and diffusion error lower than $1.0 \%$, a resolution of 8 points per time period is required.

In case of damped waves $\operatorname{Im}(\widetilde{\omega}) \neq 0$, the numerical error properties of the implicit Fractional Step method becomes even better. This is illustrated in Figure 3, where the dispersion error can become almost 10 times better in comparison to the 4th order explicit Runge-Kutta method.

\subsubsection{Space discretisation}

The discretisation in space is based on a collocated Finite Volume scheme for unstructured grids, where all unknowns are located at the nodes of the grid. The principle ideas can be found in Schneider et. al [23], [24]. The control volumes are constructed as dual boxes (see Figure 4).

The different terms related to the unknowns $q_{k}$ in the compressible Navier-Stokes equations are modelled by the Finite Volume approach:

$$
\begin{gathered}
q_{k} \Longrightarrow \int_{\Omega_{i}} q_{k} d V \approx q_{k}\left|\Omega_{i}\right|=q_{k} \sum_{i=1}^{n}\left|S C V_{i}\right| \\
\nabla q_{k} \Longrightarrow \int_{\partial \Omega_{i}} q_{k} \cdot n d \Gamma=\sum_{j=1}^{n_{i p}} \int_{\partial \Omega_{i j}} q_{k} \cdot n_{j} d \Gamma \approx \sum_{j=1}^{n_{i p}} q_{k_{j}} \cdot n_{j}\left|\partial \Omega_{i j}\right| \approx \sum_{j=1}^{n_{i p}} \sum_{c o=1}^{n_{c o}} N_{c o} q_{k_{c o}} \cdot n_{j}\left|\partial \Omega_{i j}\right| \\
\Delta q_{k} \Longrightarrow \int_{\partial \Omega_{i}} \nabla q_{k} \cdot n d \Gamma \approx \sum_{j=1}^{n_{i p}} \nabla q_{k_{j}} n_{j}\left|\partial \Omega_{i j}\right| \approx \sum_{j=1}^{n_{i p}} \sum_{c o=1}^{n_{c o}} \nabla N_{c o} q_{k_{c o}} n_{j}\left|\partial \Omega_{i j}\right|
\end{gathered}
$$

where $N_{c o}$ are bilinear ansatz functions on the element to obtain a relation between the integration point and nodal values. More details about upwinding and the treatment of the acoustic relevant terms can be found in Gordner [10].

This discretisation is only of second order for acoustic waves, which results in no diffusion, but considerable dispersion errors, see i.e. Tam and Webb [29] and the lines in Figure 5 for $\kappa=1.0$. 

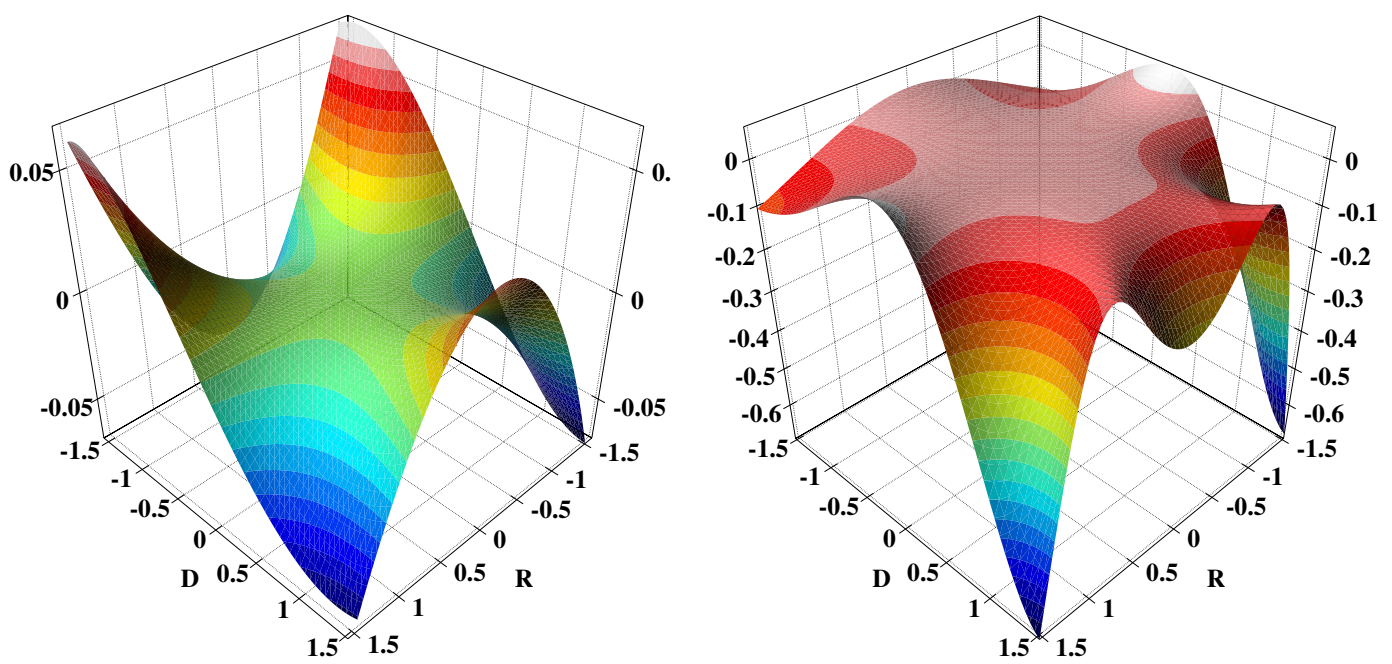

Figure 3. Comparison of the numerical damping errors of the Fractional Step method (left) compared to the standard explicit 4th order Runge-Kutta scheme(right) for waves with non zero imaginary part. $D$ denotes the imaginary part, while $R$ indicates the real part of $\widetilde{\omega} \Delta t$. $D>0$ denotes a damped wave.

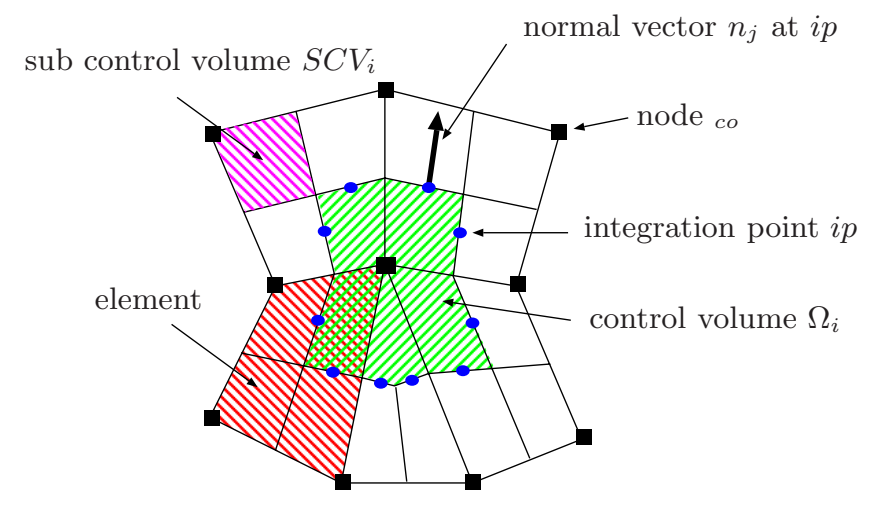

Figure 4. Collocated Finite Volume grid.

Varying grid sizes of an unstructured grid influence the numerical dispersion and damping errors of the solution. If the grid size is reduced by a factor of two in the direction of the travelling acoustic wave $\kappa=\frac{h_{i+1}}{h_{i}}=$ 0.5 additional damping and less dispersion is introduced, see Figure 5 . If the grid size is continously increased by a factor of $2(\kappa=2.0)$, the wave become instable. The imaginary part then becomes positive, which is illustrated in Figure 5. Hence, the grid size has to be designed with a smooth transition from the fine near field to the coarser far-field.

Since a 4th order time discretisation is used, it is desirable to increase the accuracy of the space discretisation at least to the same order. Doing this in a direct way for the described Finite Volume method on a collocated grid is a tremendous computational effort. If one assumes a smooth solution, the order and accuracy of the solution can be increased by an extrapolation technique. Since an implicit time discretisation is used, an algebraic system of equation has to be solved in any case. A geometric multigrid solver can be adjusted to be robust 


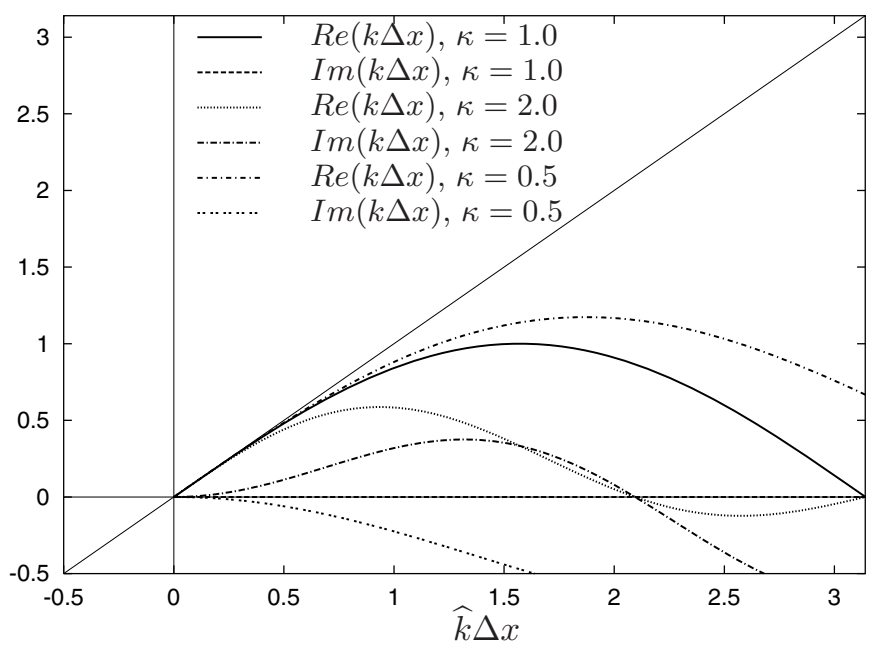

Figure 5. Numerical diffusion and dispersion error of the Finite Volume approach with varying grid sizes. $\kappa=1.0$ denotes a constant grid size. $\kappa=\frac{h_{i+1}}{h_{i}}=0.5$ describes a grid size reduction in direction of the travelling wave by a factor of 2 , while $\kappa=2.0$ denotes a grid size doubling in moving direction.

against the Mach number, so that the convergence of the scheme is independent of the Mach number and does not deteriorate for $M \rightarrow 0$, see Gordner [10]. Beside this, multigrid can be used as well to increase the order of the solution by $\tau$-extrapolation. This technique was first introduced by Brandt [5] and further developed by Bernert [2] and Fulton [8]. Rüde and Jung [19] have shown the applicability to unstructured grids.

In the following the idea of $\tau$-extrapolation is described very briefly for a two-grid algorithm to show the basic principles. However, the idea can be transfered to a multigrid procedure as well. Further details about multigrid methods can be found in Hackbusch [12] or Wesseling [30].

Within the multigrid process, a linear algebraic system of equations of the form

$$
J_{l} q_{l}=b_{l}, \quad J_{l} \in \mathbb{R}^{n} \times \mathbb{R}^{n}, \quad q_{l}, b_{l} \in \mathbb{R},
$$

has to be solved, where $l$ denotes the grid level with a certain mesh size $h_{l}$. After some relaxation steps on the fine grid, a coarse grid correction is determined on the next higher grid level with mesh size $h_{(l-1)}>h_{l}$ by

$$
c_{l-1}=J^{-1} R_{l}^{l-1}\left(b_{l}-J_{l} q_{l}\right)=J^{-1} R_{l}^{l-1} d_{l} .
$$

$R_{l}^{l-1}$ denotes the grid transfer (restriction) of the fine grid defect $d_{l}$ to the next coarser grid. This coarse grid correction $c_{l-1}$ is then interpolated to the fine grid and added to the fine grid solution $q_{l}$.

The order of the coarse grid correction $c_{l-1}$ depends on the order of the right hand side defect $d_{l-1}=R_{l}^{l-1} d_{l}$, and one can increase the order of the coarse grid solution by increasing the order of the right hand side $d_{l-1}$. This can be done by a Richardson-type extrapolation technique, where the right hand side on grid level $l-i$ is a combination of different right hand sides of finer grid levels

$$
d_{l-i}^{*}:=\sum_{l=h}^{l-i} c_{l} R_{l}^{l-i} d_{l} .
$$

The order of the assembled right hand sides $d_{l}$ are $O(n)$. Regularly, the order is the same on all grid levels, since the same discretisation is used on all levels. The coefficients $c_{l}$ depend on the order of the assembled right 
hand side $d_{l}$ and on the mesh size ratios. The used coefficients are given in Table 1 . Here, the finer grid levels are derived from the coarser grids by regular refinement. The basic principles to derive the coefficients can be found i.e. in Ferziger [7] or Stoer [25].

\begin{tabular}{c|ccc} 
& $c_{3}$ & $c_{2}$ & $c_{1}$ \\
\hline $\mathrm{l}=2$ & - & $-\frac{1}{3}$ & $\frac{4}{3}$ \\
$\mathrm{l}=3$ & $-\frac{3}{45}$ & $-\frac{16}{45}$ & $\frac{64}{45}$
\end{tabular}

TABLE 1. Coefficients for the $\tau$-extrapolation procedure over two or three levels.

In the multigrid solution process, the $\tau$-extrapolation is done on a subset of multigrid levels, just in order to reach the desired accuracy in space. This is illustrated in Figure 6. After one $\tau$-extrapolated multigrid V-cycle with a extrapolated right hand side, a couple of regular multigrid V-cycles are performed to solve the system. Hereby, the order of the solution is preserved and not further increased.

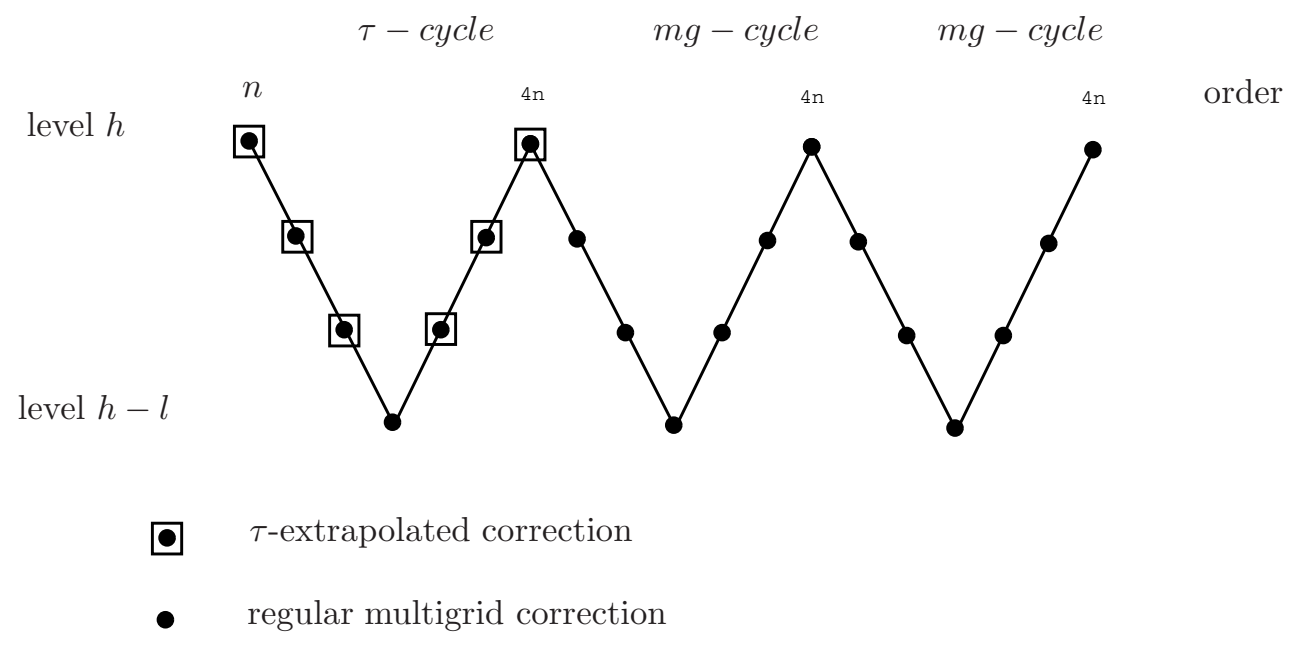

Figure 6. A 4 level linear multigrid procedure with one full $\tau$-extrapolated cycle. After a 3 level $\tau$-extrapolated multigrid cycle, the order is increased from $n$ to $4 n$ and stays the same during the following regular multigrid cycles.

\section{Numerical EXAmple}

\subsection{Running wave}

The simple example covers an acoustic wave generated at normalised time $t=0$ at the duct outflow, that travels in upstream direction. The viscous terms are set to zero, which makes it easier to find an analytical solution to compare with. The normalised wave length is $\lambda=1.5$ with $\omega \approx 4.188$. The Mach number is $M=10^{-3}$ and the pressure amplitude of the superimposed sinus-shaped acoustic wave is $p^{\prime}=2 \cdot 10^{-6}$, which is small in comparison to the normalised mean pressure $p=1.0$. At $t=1.5$ the exact solution can be compared with the numerical solution, in order to identify numerical damping and dispersion. For simplicity, the grid is equally spaced and the mesh size is fine enough -(80 points per wave length)- such that the numerical errors of 


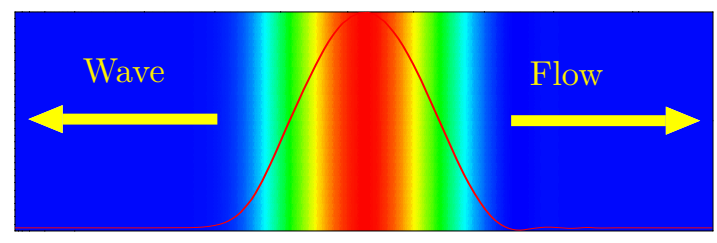

FiguRE 7. Travelling pressure wave configuration in a pipe flow.

the space discretisation are negligible small. Numerical dispersion will lead to a modified wave velocity, while numerical damping would decrease the amplitude of the wave.

The numerical damping an dispersion properties of the time and space discretisation are depending on the $\omega \Delta t$ or $\lambda h$ respectively. For a given angular frequency $\omega$ or wave length $\lambda$, the values $\omega \Delta t$ and $\lambda h$ determine how many points are used to model a time period or wave length. (I.e $\omega \Delta t=1.0$ means a resolution of 8 points per time period.) According to equations (28) and (29) the numerical error depends on $\omega \Delta t$ and $\lambda h$ respectively and can be controlled with the number of grid points per time period or wave length.

Based on the theoretical analysis of the last chapter, the resolution for the time and space discretisation is chosen to guarantee numerical dispersion and damping errors less than $1.0 \%$. This is to obtain a reasonable long time behaviour of the running acoustic wave. A detailed analysis and comparison of different discretisation methods and their numerical properties is beyond the scope of this paper. The running wave example is used to verify and demonstrate the theoretical results of the last chapter.

In Figure 8 at $t=1.5$, the position of the wave peak, expected to be at the normalised position $x=-0.25$, and the amplitude of the numerical solution in comparison to the exact solution is given. The numerical damping and dispersion properties of the time discretisation depend on $\omega \Delta t$. For $\omega \Delta t<1.0$, which means a resolution of 8 points per time period, the numerical damping and dispersion errors are lower than $1.0 \%$. A desired dispersion error of $0.1 \%$ would require $\omega \Delta t<0.15$, hence approximately 50 points per time period. Increasing $\omega \Delta t$ to 2.0 will lead to higher numerical errors as can be recognised in Figure 8 and Figure 2. After approximately one time period $t=1.5$ with a Mach number $M=0.001$ the wave has to travel an exact distance of 1.4985 , while the numerical solution travels a distance of 1.477 for $\omega \Delta t<1.0$, which is a numerical error of $\approx 1.5 \%$. This corresponds fairly well with the theoretical investigations and one can derive reasonable predictions for the long term behaviour of the wave. A more detailed analysis can be found in Gordner [10].

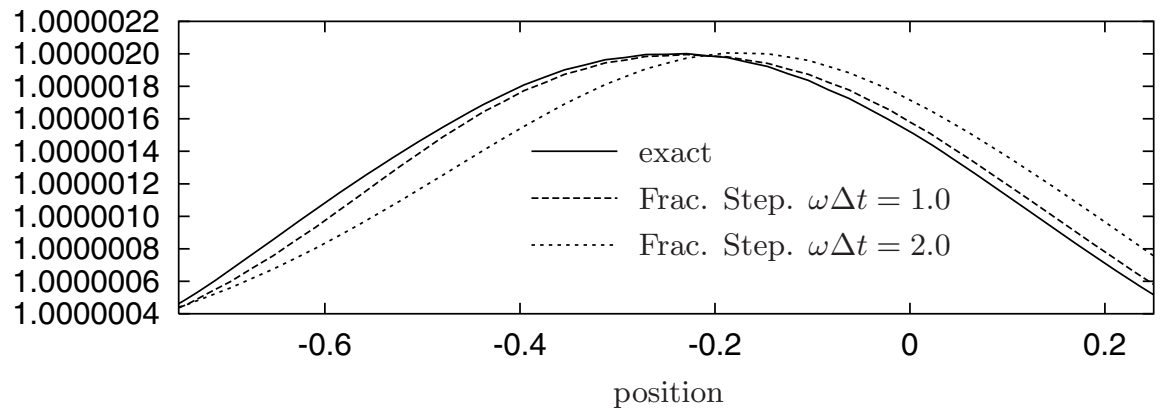

FiguRE 8. Numerical diffusion and dispersion errors for the Fractional-Step time discretisation.

The same numerical experiment can be made with different grid sizes $h$ to test the accuracy of the space discretisation. For second order central schemes only dispersion errors are expected. This is illustrated in 


\begin{tabular}{c|c} 
& mesh size \\
\hline Level 4 & $\lambda h=0.1$ \\
Level 3 & $\lambda h=0.2$ \\
Level 2 & $\lambda h=0.4$
\end{tabular}

TABLE 2. Mesh sizes for different grid levels. The grids are constructed with hierarchical grid refinement.

Figure 9. Here, the numerical diffusion and dispersion errors of two different grid sizes with $\lambda h=0.2$ and 0.4 are compared with the analytical solution.

Since the Finite Volume approach is only of second order, approximately 20 points per wave length, or $\lambda h<0.4$ are needed to guarantee numerical errors less than 1.0\%. A detailed analysis can be found in Gordner [10] as well.

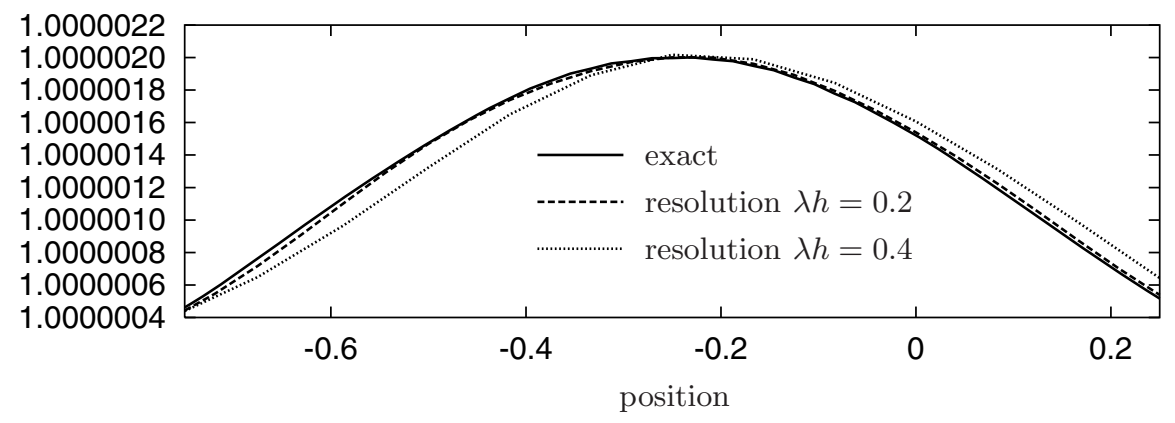

Figure 9. Numerical diffusion and dispersion errors for the Finite Volume space discretisation.

A possibility to reduce the number of points needed per wave length is increasing the order of the spatial discretisation. There are several specialised Finite Difference scheme of 6th or even higher orders, i.e. DRPschemes, see Tam \& Webb [29]. Another possibility is using higher order Discontinuous Galerkin (DG) schemes, i.e Munz [17] and Cockburn et. al. [6] for basic principles. However, these schemes and higher order Finite Volume schemes are costly with respect to the numerical assembling effort. As proposed in the last chapter, an alternative way for increasing the order of the numerical solution is a $\tau$-extrapolated multigrid procedure. Again, the travelling wave configuration is taken to verify the theoretical investigations. A 2- or 3- level $\tau$ extrapolated multigrid solution should considerably reduce the dispersion error of the acoustic wave. This is illustrated in Figure 10, where the position of the wave peak is compared for different mesh sizes, see Table 2

Note that there is a diffusion error inherent in the solution, since the time step size was chosen larger to reduce computational time, which does not influence the principle results.

As expected, the dispersion error increases for increasing mesh size (see the curves for Level 4, Level 3 and Level 2 in Figure 10). With a 2-level or 3-level $\tau$-extrapolated solution on Level 2, the numerical dispersion error can be considerably reduced, so that almost no difference in the wave peak position in comparison to the Level 4 solution is detected. Here, the computational costs are reduced, while preserving the same magnitude of numerical errors.

\subsection{Trailing edge bluntness noise}

In section 2 the number of unknowns have been estimated, that can be saved using an unstructured grid. This is verified with another numerical experiment. Here, trailing-edge thickness noise of a flat plate with $0.25 \%$ thickness at a Mach number $M=0.2$ is simulated. The stress tensor is set to zero, $\tau=0$, in order to eliminate any acoustic sources based on viscosity effects. Non-reflecting b.c. are based on the ideas, found in 


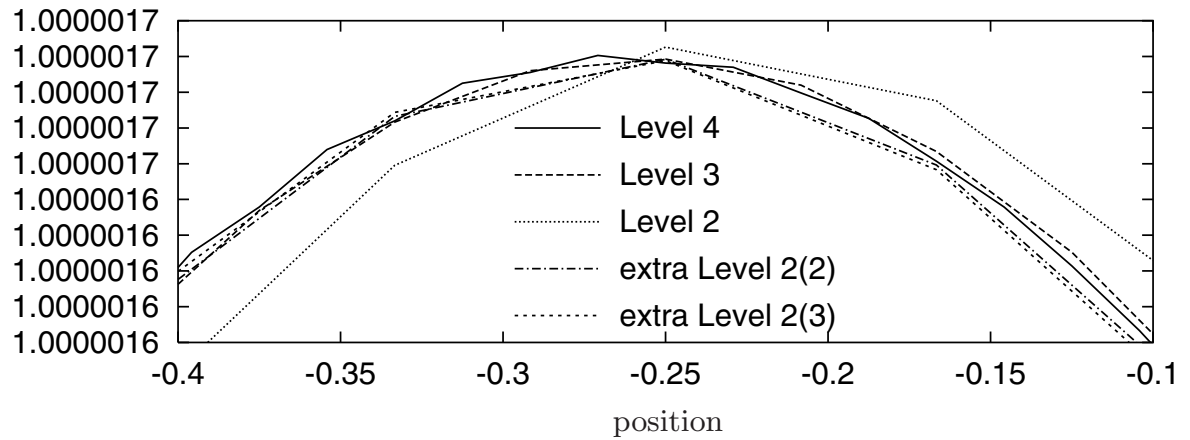

Figure 10. Comparison of the $\tau$-extrapolated versus regular multigrid solution. The numerical dispersion error is characterised by a phase shift of the wave peak.

\begin{tabular}{lrrlr}
\hline & theo. $\frac{\# n(M=1)}{\# n(M)}$ & $m$ refinements & $\# n$ & real $\frac{\# n(M=1)}{\# n(M)}$ \\
\hline equally spaced grid & - & - & $\sim 10^{9}$ & - \\
$M=0.1$ & 100 & 9 & $\sim 4 \cdot 10^{7}$ & 25 \\
$M=0.005$ & 40000 & 5 & $\sim 10^{6}$ & 1000 \\
\hline TABLE 3. Grid points needed on unstructured grids versus equally refined grid.
\end{tabular}

Selle et al. [22], Poinsot et al. [20] and Polifke et al. [21]. The approach introduces additional stiffness, instead of damping terms used within a PML approach, introduced by Berenger [1].

It is expected that the flow structures near the plate wall and the trailing and leading edge of the plate, are smaller than those in the acoustic far-field. Hence, the region around the trailing and leading edge of the flat plate, the near flow field, is much finer resolved than the acoustic far-field.
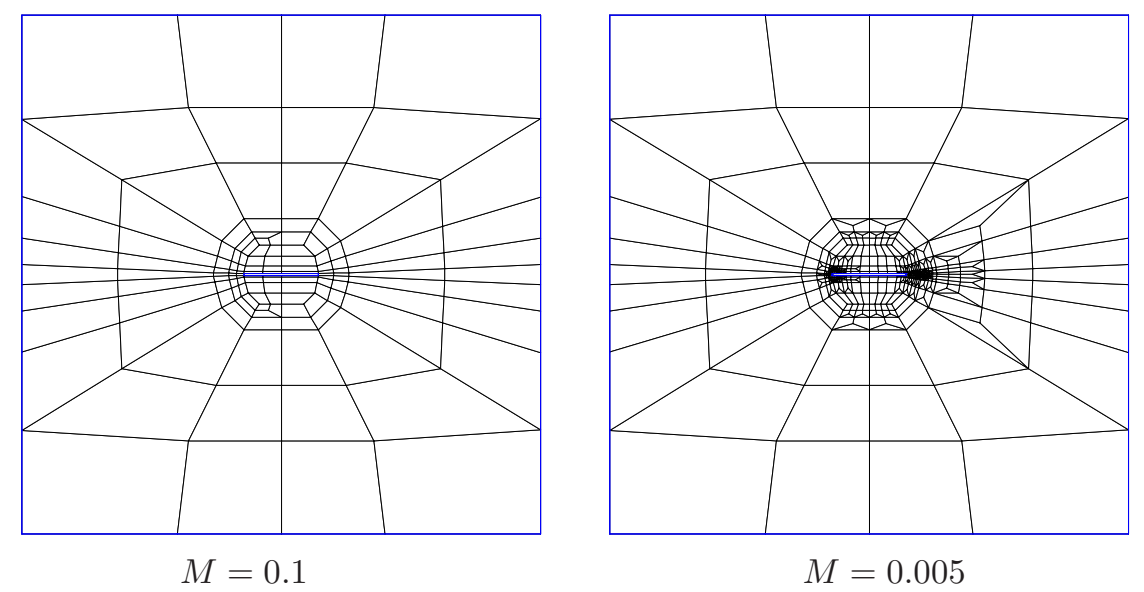

Figure 11. Computational domain on grid level 0 for different Mach numbers.

The unstructured grids on level 0 of the multigrid scheme for two different Mach numbers $M$ are plotted in Figure 11. The grids for the simulation are much finer and are obtained by $m$-times regular refinement, such that $h_{\min } \approx 4 \cdot 10^{-4}$. The value $m$ and the number of grid points of the finest grids are illustrated in Table 3 . The comparison between the theoretical grid point reduction and the real gained benefit reveals a fairly good 
correspondence, with a constant $C \approx\{4, . ., 40\}$. Hence, especially for $M=0.005$ the grid is still too fine in the far-field.

The pressure solution at $t=7500$ for a Mach number $M=0.2$ is given in Figure 12. The acoustic far-field generated by the shear layer behind the trailing-edge of the flat plate, its directivity and the shear layer itself, can be recognised. The maximum and minimum CFL-number on the grid are

$$
C F L_{\text {min }} \approx 0.02, \quad C F L_{\text {max }} \approx 4
$$

Here, the maximum CFL-number is in good agreement with the theoretical investigations in equation (17), for $C_{G} \approx 1$, while the far-field mesh size is still too fine for an evenly distributed numerical error. This indicates the small minimum CFL-number which should be of the order of $C_{G}$.
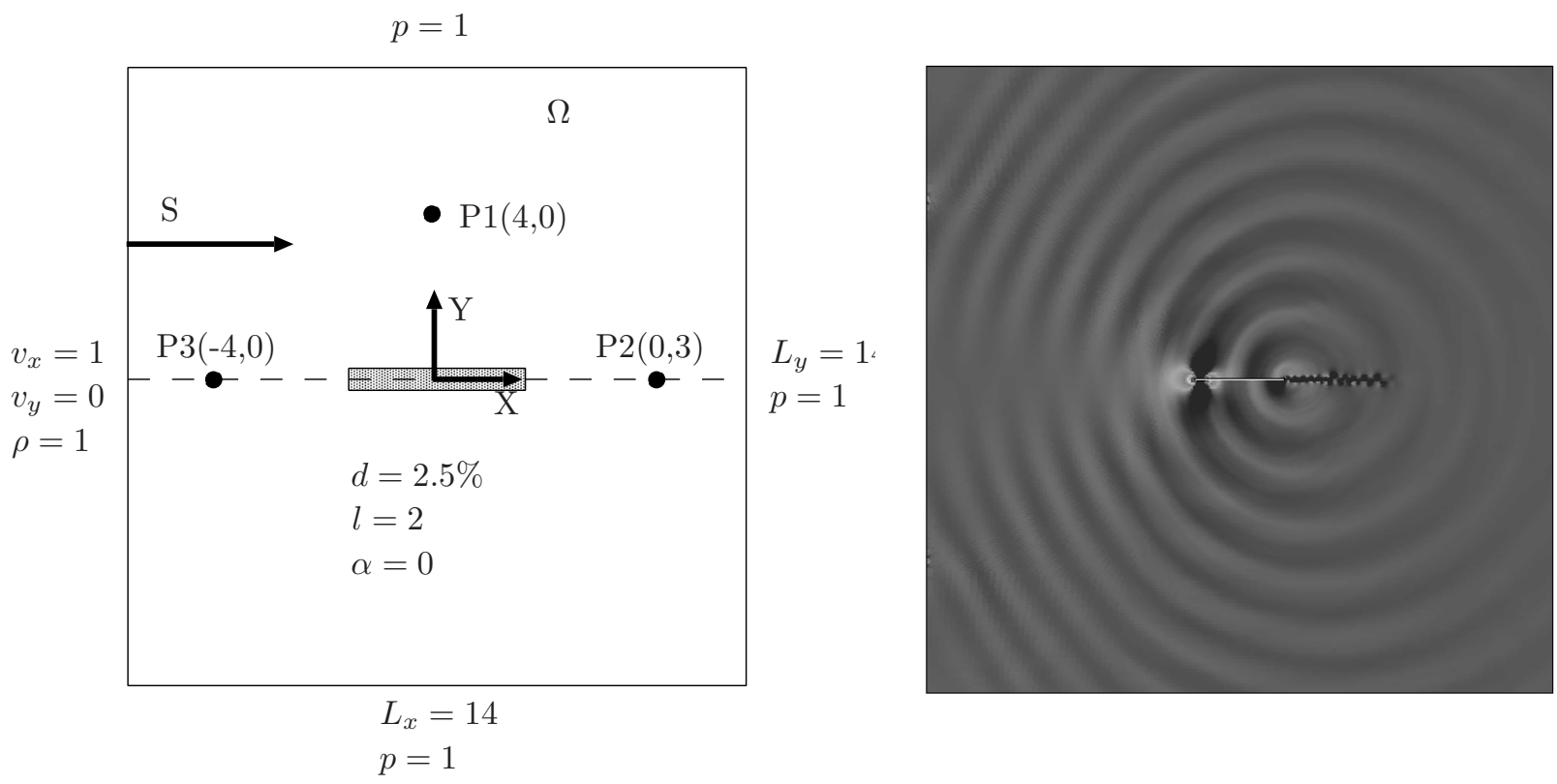

Figure 12. Geometrical configuration (left) and Pressure field (right) of the trailing edge noise simulation. The shear layer at the trailing edge of the flat plate generates acoustic pressure fluctuations, that evoke acoustic waves in the far-field.

In Figure 13 the pressure variations at three different points are illustrated. The positions of the points are illustrated in Figure 12. One is located in front of the leading edge, one behind the trailing edge and the third one is perpendicular to the plate chord. The corresponding frequency spectra are plotted in Figure 14

\section{Conclusions}

With the use of an unstructured grid, where the local mesh size is adjusted to the local flow situation such that the numerical discretisation error in space is equally distributed on the domain $\Omega$, the number of unknown can be reduced considerably. The benefit, which scales $\sim C \frac{1}{M^{d}}$, is Mach number and dimension dependent. For small Mach numbers $M<<1$, due to the stiffness of the problem and the unstructuredness of the grid, explicit time discretisations would require unnecessarily small time step sizes $\sim M$. An effort, that does not pay-off in the accuracy of the solution. 


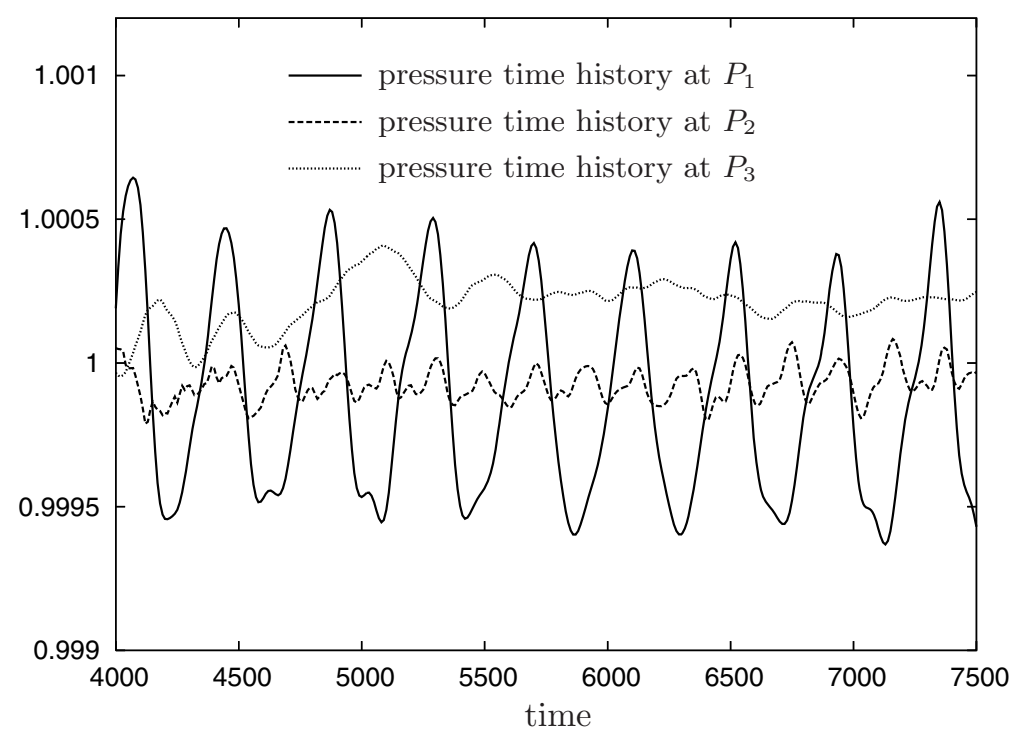

Figure 13. Time history of the normalized pressure fluctuations at the points $P_{1}, P_{2}$ and $P_{3}$.

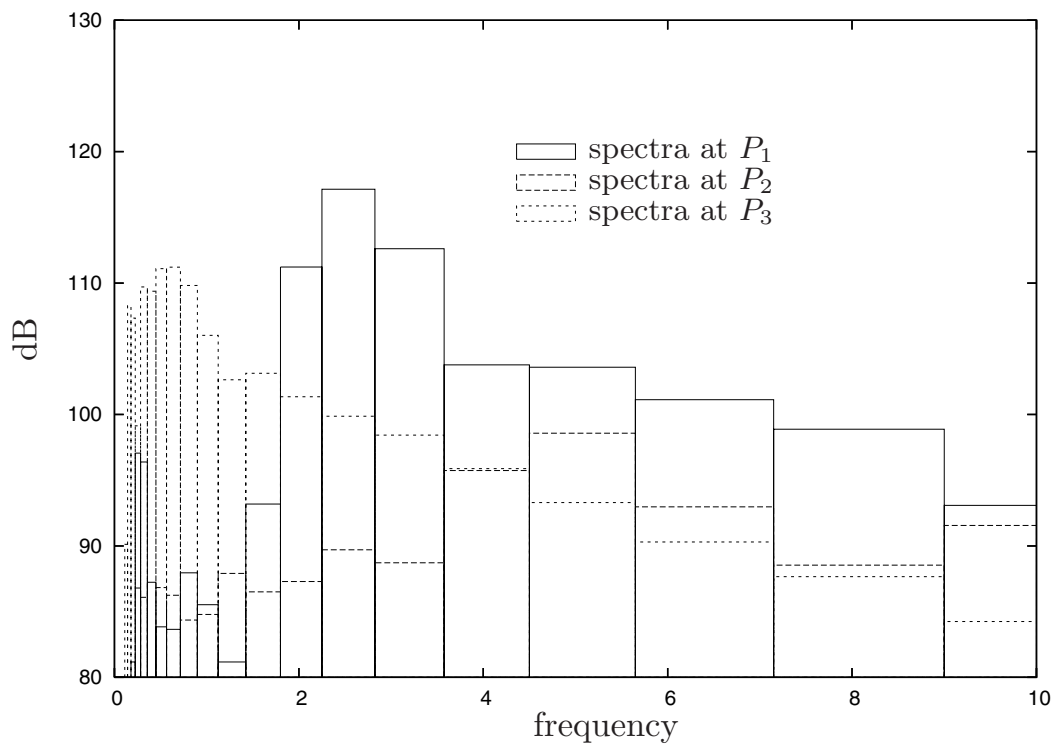

Figure 14. Frequency spectra of the pressure signal at the points $P_{1}, P_{2}$ and $P_{3}$.

Implicit time discretisations can handle CFL-numbers larger than 1 and can sustain an evenly distributed numerical error on the highly unstructured grids, if the order of the time discretisation is of the same order as the space discretisation or even better. However, as a drawback, it is required to solve an algebraic system of equation efficiently. A geometrical multigrid solver demonstrates good performance in two respects. First it solves the algebraic system of equations robust against the Mach number, such that the convergence rate is Mach number independent. On the other hand, the multigrid procedure can be used as well to increase the 
accuracy of the numerical solution by the $\tau$-extrapolation technique. Hereby, the computational cost can be further reduced, while the accuracy is retained or even improved.

\section{REFERENCES}

[1] J. P. Berenger, A perfectly matched layer for the absorption of electromagnetic waves, Journal of Computational Physics, 114, 1994.

[2] K. Bernert, $\tau$-Extrapolation - Theoretical Foundation, Numerical Experiment, and Application to Navier-Stokes Equations, SIAM. Journal of Scientific Computing, Vol. 18, No. 2, pp. 460-478, 1997.

[3] W.K. Blake, Mechanics of Flow-Induced Sound and Vibration; Vol I: Basic General Concepts and Elementary Sources, and Vol II: Complex Flow-Structure Interaction. New-York, Academia Press.

[4] H. Bijl and P. Wesseling, A Unified Method for Computing Incompressible and Compressible Flows in Boundary-Fitted Coordinates, Journal of Computational Physics, 141, 153-173, (1998).

[5] A. Brandt, Multi-Level adaptive solutions to boundary value problems., Mathematics of Computation, Vol. 31, pp. 333-390, 1977.

[6] B. Cockburn, G. E. Karniadakis, and C. Shu, editors. Discontinuous Galerkin Methods: Theory, Computation and Applications, volume 11 of Lecture Notes in Computational Science and Engineering. Springer-Verlag, Berlin, Heidelberg, New York, 2000.

[7] J. H. Ferziger, Numerical Methods for Engineering Application, 1. edition, Wiley, 1981.

[8] S.R. Fulton, On the accuracy of multigrid truncation error estimates, Electronic Transactions on Numerical Analysis,Vol. 15, pp. 29-37, 2003.

[9] R. Glowinski and J. Periaux, Numerical Methods for nonlinear Problems in Fluid Dynamics. In: Proceedings International Seminar on Scientific Supercomputers, Paris, (1987).

[10] A. Gordner. Numerische Simulation nichtlinearer Aeroakustik bei kleinen Machzahlen. PhD thesis, University of Heidelberg, (2005).

[11] H. Guillard and C. Viozat, On the behaviour of upwind schemes in the low Mach number limit, Computers and Fluids, 29, 63-86, 1999.

[12] W. Hackbusch, Multi-Grid Methods and Applications. Springer Verlag, 1985.

[13] P. Költzsch, Strömungsakustik - eine aktuelle Übersicht. In: Bericht ET-IAS-01-2000 Technische Universität Dresden. (Eds. Der Rektor), (2000), 1-52.

[14] S.K. Lele, Computational Aeroacoustics: A Review. AIAA paper 97-0018, (1997), 1ff.

[15] J. F. Lynn, B. van Leer and D. Lee, Multigrid solution of the Euler Equations with Local Preconditioning, International Conference on Numerical Methods in Fluid Dynamics, Monterey, Ca, 1996.

[16] S. Müller-Urbaniak, Eine Analyse des Zwischenschritt- $\Theta$-Verfahrens zur Lösung der instationären Navier-Stokes-Gleichungen. $\mathrm{PhD}$ thesis, University of Heidelberg, (1993).

[17] C.-D. Munz and E. Krämer, Verfahren hoher Ordnung in der numerischen Aeroakustik und Str"omungsmechanik, Presentation at the ERCOFTAC Technologietag, 30th September 2005, University of Stuttgart.

[18] S. Roller, T. Schwartzkopff, R. Fortenbach, M. Dumbser and C.-D. Munz, Calculation of Low Mach Number Acoustics: A Comparison of MPV, EIF and Linearized Euler Equations, ESIAM: Mathematical Modelling and Numerical Analysis, M2AN, Vol. 39, no. 3, 561-576, 2005.

[19] M. Jung and U. Rüde, Implicit Extrapolation Methods for variable Coefficient Problems, SFB-report Nr. 342/24/95 A, 1995.

[20] T.J. Poinsot and S.K. Lele, Boundary Conditions for Direct Simulations of Compressible Viscous Flows, Journal of Computational Physics, vol. 101, 104-129, 1992.

[21] W. Polifke and C. Wall, Non-Reflecting Boundary Conditions for Acoustic Transfer Matrix Equations with LES, Standford University. Center for Turbulence Research, Proceedings of the Summer School 2002, 1ff, 2002.

$[22]$ L. Selle, F. Nicoud and T.J. Poinsot, Actual Impedance of Nonreflecting Boundary Conditions: Implications for Computation of Resonators, AIAA Journal, vol. 42 No. 5, 958-964, 2004.

[23] G.E. Schneider and M.J. Raw, Control Volume finite-element method for heat transfer and fluid flow using colocated variables - 1. Computational procedure, Numerical Heat Transfer, 11, 363-390, 1987.

[24] G.E. Schneider and S.M.H. Karimian, Pressure based Computational Method for Compressible and Incompressible Flows, Journal of Thermophysics and Heat Transfer, vol. 8(2), 267-274, 1994.

[25] J. Stoer, Numerische Mathematik 1, Springer 9.edition, 2005.

[26] S. Turek, Efficient Solvers for Incompressible Flow Problems. An Algorithmic and Computational Approach. Springer, 1999.

[27] E. Turkel, Preconditioning Techniques in Computational Fluid Dynamics, Ann. Rev. of Fluid Mechanics, 31, 385-416, 1999.

[28] E. Turkel and V. N. Vatsa, Local Preconditioners For Steady and Unsteady Flow Applications, ESIAM: Mathematical Modelling and Numerical Analysis, M2AN, Vol. 39, no. 3, 515-535, 2005.

[29] C.K.W. Tam and J.C. Webb, Dispersion-Relation-Preserving Finite Difference Schemes for Computational Aeroacoustics, Journal of Computational Physics, Vol. 107, pp. 262-281, 1993.

[30] P. Wesseling, An Instroduction to Multigrid Methods,2. edition, Teubner, 1993. 